\title{
ORIGINAL
}

\author{
I. Muench • A. Renuka Balakrishna • J. E. Huber
}

\section{Periodic boundary conditions for the simulation of 3D domain patterns in tetragonal ferroelectric material}

Received: 5 January 2018 / Accepted: 5 June 2018 / Published online: 18 June 2018 (C) The Author(s) 2018

\begin{abstract}
Periodic domain patterns in tetragonal ferroelectrics are explored using a phase field model calibrated for barium titanate. In this context, we discuss the standard periodic boundary condition and introduce the concept of reverse periodic boundary conditions. Both concepts allow the assembly of cubic cells in accordance with mechanical and electrical conditions. However, application of the reverse periodic boundary condition is due to an increased size of the RVE and enforces more complex structures compared to the standard condition. This may be of particular interest for other multiphysics simulations. Additionally, we formulate mechanical side conditions with minimal spherical (hydrostatic) stress, or conditions with controlled average strain. It is found that in sufficiently small periodic cells, only a uniform single domain, or the simplest stripe domains constitute equilibrium states. However, once the periodic cells are of order 20 domain wall widths in size, more complex, 3-dimensional patterns emerge. Some of these patterns are known from prior studies, but we also identify other domain patterns with long, ribbon-like domains threaded through them and some vortex-like structures.
\end{abstract}

Keywords Periodic boundary conditions · RVE simulation - Tetragonal ferroelectrics · Polarization patterns · Polarization vortex $\cdot$ Multiphysics simulations

\section{Introduction}

Tetragonal ferroelectrics such as barium titanate, $\mathrm{BaTiO}_{3}$, are characterized by a spontaneous electric polarization lying parallel to the pseudo-cubic axes of the crystal. On cooling $\mathrm{BaTiO}_{3}$ through the Curie temperature, $T_{c} \cong 120^{\circ} \mathrm{C}$, the material gains a spontaneous polarization and transforms its crystal structure from the cubic, paraelectric to the tetragonal, ferroelectric state. This phase transformation induces lattice strains and, depending on the surrounding constraints, it causes deformation or internal mechanical stress [1,2]. During the phase transformation, regions with uniform electric polarization group together, forming domains separated by narrow domain walls.

I. Muench

Karlsruhe Institute of Technology (KIT), Institute for Structural Analysis, Karlsruhe, Germany

E-mail: ingo.muench@kit.edu

A. Renuka Balakrishna

Department of Materials Science and Engineering, Massachusetts Institute of Technology (MIT), Cambridge, MA 02139, USA

E-mail: ananyarb@mit.edu

J. E. Huber $(\varangle)$

Department of Engineering Science, University of Oxford, Oxford OX1 3PJ, United Kingdom

E-mail: john.huber@eng.ox.ac.uk 
According to Maxwell's equations, domains usually arrange with electrically compatible 'head-to-tail' polarization, which minimizes energy due to charged domain walls or strong compensating electric fields $[3,4]$. Similarly, domain patterns minimize internal mechanical stress through compatible arrangements of spontaneous strain [5]. This results in the well-known $90^{\circ}$ and $180^{\circ}$ domain walls in tetragonal ferroelectrics.

Energy minimization provides a powerful method for understanding how domain patterns form. Since the domain walls are typically less than a few $\mathrm{nm}$ in thickness, a sharp interface approximation is attractive [6]. From this approach and from observation of micro- or nano-structure, many domain patterns have been identified [3,5, 7-10]. The simplest known arrangements are bundles of $90^{\circ}$ and $180^{\circ}$ domains forming laminates of alternating stripes. In thin films or lamellae, the domain bundles are commonly polarized in-plane [11]. However, thicker plates or bulk materials can form energy minimizing patterns with domains polarized in all three axial directions $[3,12]$. The formation of periodic or nearly periodic patterns of domains is commonly observed; the effect of specimen size and mechanical strain on the formation of such periodic domain patterns has also been widely reported $[4,12-15]$. The spatial distribution of domains affects ferroelectric properties at both fine scale and macroscale $[8,11,16-21]$. Rödel [21] discussed the dependence of piezoelectric coefficients on the volume fractions of domains, while Weng and Wong [22] showed that the macroscale ferroelectric behaviour is enhanced in certain laminated domain patterns. There is great interest in the domain patterns because of the potential to exploit engineered configurations of domains for energy harvesting, actuation, sensing, and memory devices [23-27].

Significant progress in understanding domains has been made with relatively simple models. However, results based on a sharp interface approach neglect the details of domain walls and their interactions, which become important when the size of domains is comparable to the domain wall width. In such cases, the formation of domain patterns and their stability has not been fully explored. Phase field models, with an order parameter that varies across the domain walls, offer an opportunity to study nanoscale domain patterns with a physically realistic representation of domain wall energy, bulk electromechanical energy, and the effects of external electromechanical loading. The use of phase field models for ferroelectric domains is well-established [28-33]. Several studies explore the evolution of polarized domains in thin films [34-37], under the effect of electromechanical loads. These studies explore the local effects of substrate strain on domain shapes [36], domain wall movement [34] and the coercive field [37]. In ferroelectric-bulk, the 3-dimensional effect of the electromechanical boundary conditions influences the formation of equilibrium domain patterns. 3D periodic laminates with microstructural features such as curved domain walls [5,15] and ribbon-shaped domains [9] have been experimentally observed. These 3D microstructural features evolve under external electromechanical loads and influence the nanoscale behaviour of ferroelectrics [9]. Exploring the spatiotemporal evolution of periodic polarization patterns in a theoretical framework would indicate the complexities in pattern formation and provide initial steps towards nanoscale experimentation. Recently, we used the same phase field model as in this work to explore nanoscale domain patterns [14]. Starting from the patterns predicted by energy minimization with sharp interfaces [5], it was found that several of the patterns dissolve into simpler, more stable arrangements such as single domains or alternating stripes. For computational speed, the calculations were mainly carried out using 2D models that limit the freedom of the system to relax into fully 3D domain patterns. Extending the study to consider periodic volumes large enough to support domain patterns makes heavy demands on computation, and the calculations reported in the present work typically took days to weeks each, running as parallelized code using 32 cores on an Intel Xeon CPU at $3.2 \mathrm{GHz}$.

The goal of this work is to establish and explore two kinds of periodic boundary conditions for scalar and vectorial fields at the bound of representative volume elements (RVE). This is motivated by the fact that periodic boundary conditions generally restrict the solution space. Thus, any RVE simulation may benefit from this discussion since our model demonstrates that differing periodic boundary conditions naturally lead to an enriched variety of periodic domain patterns. The principle of the reverse periodic boundary condition is an enhanced assembly rule, where we superpose translation and rotation.

We study a range of periodic cell sizes and then focus on a size just sufficient to allow complex domain patterns to form. Such complex patterns could be useful in memory applications where arrays of writable domains and skyrmion-like topologies [38] are of current interest. Periodic patterns may be artificially enforced by imposing a template or may arise naturally through minimization of energy. Nature, of course, has available any periodic cell size or none. The exact reason why nearly periodic patterns of domains commonly form is not known. It may be that similar conditions of stress or cooling rate during the phase transition favour the nucleation of particular domains that then adjust into a nearly periodic form to minimize energy. More likely, 
a small region of pattern first forms and then becomes the template for surrounding regions which copy the pattern. We show that cell sizes of at least tens of domain wall widths are necessary in order to support domain patterns other than simple stripes or single domains.

Further, we explore the effect of strained states on the formation of domain patterns. The study is motivated by a classification of low-energy periodic domain patterns given by Tsou et al. [5] in which several families of periodic domain patterns were identified. We employ a 3-D phase field model $[39,40]$, which is calibrated for barium titanate and reproduces the transition from an initial paraelectric state to the tetragonal ferroelectric state at the Curie point, whereupon domain patterns evolve to minimize internal energy. We do not force any particular pattern by imposing a template or by initial conditions: instead, the simulations take a randomly perturbed initial state and relax this towards equilibrium. Since it is impractical to simulate 3-dimensional regions much larger than a few tens of $\mathrm{nm}$ in size, we make use of periodic boundary conditions, which allow the assembly of simulated volumes into larger periodic cells. Additionally, the simulations are carried out with controls on the average strain, introduced as various side conditions. This allows for the effect of a remanent ferroelastic strain, appearing at the phase transition from the paraelectric to the ferroelectric state.

The paper is organized as follows: the underlying model is given in Sect. 2, followed by a discussion of periodic boundary conditions in Sect. 3. The results of our simulations are presented and discussed in Sect. 4, followed by conclusions in Sect. 5 .

\section{The material model}

The model follows the Landau-Devonshire theory of ferroelectrics [41], the work of Fried and Gurtin [42,43], and the subsequent work of Landis [39,40]. Fuller details concerning the normalization of physical fields, phase field modelling, and finite element technique are given in $[25,44,45]$. The evolution of domain patterns follows the time dependent Ginzburg-Landau equation [39], with polarization $\mathbf{P}$ as the order parameter:

$$
\operatorname{Div}\left(\frac{\partial \Psi}{\partial \operatorname{Grad}[\mathbf{P}]}\right)-\frac{\partial \Psi}{\partial \mathbf{P}}=\beta \dot{\mathbf{P}}, \quad \beta \geq 0,
$$

where $\Psi$ is the Helmholtz free energy of the system and $\beta$ is a polarization viscosity introduced to represent the dissipation associated with domain wall motion. Domains of homogeneous electric polarization evolve during the simulations, and the polarization rate on the right hand side of Eq. (1) vanishes in the final state of equilibrium.

The Helmholtz free energy includes contributions due to mechanical strain $\boldsymbol{\varepsilon}$, electric polarization $\mathbf{P}$, and electric field $\mathbf{E}$ :

$$
\Psi=\Psi_{\text {mech }}(\boldsymbol{\varepsilon}, \mathbf{P})+\Psi_{\text {well }}(\mathbf{P})+\Psi_{\text {grad }}(\operatorname{Grad}[\mathbf{P}])+\Psi_{\text {elec }}(\mathbf{P}, \mathbf{E}) .
$$

The free energy function used includes six energy wells corresponding to the six polar variants of the tetragonal ferroelectric. The curvature of the wells is calibrated to reproduce the elastic, piezoelectric, and dielectric behaviour of the individual domains, and the gradient term reproduces $90^{\circ}$ and $180^{\circ}$ domain walls with widths and energies consistent with other studies $[39,46,47]$. The model does not explicitly include flexoelectric effects, though gradients in strain are indirectly coupled to polarization through the gradient terms in the model. Simulations are initiated with $\mathbf{P} \approx 0, \boldsymbol{\varepsilon} \equiv 0$ and $\mathbf{E} \equiv 0$ throughout the simulated region. Strain controls, where used, are switched on after the first iteration of the Newton-Raphson solution scheme. The overall internal energy $\Pi=\int_{\mathcal{B}_{c}} \Psi \mathrm{d} V$ reduces as the simulation proceeds, eventually stabilizing at an equilibrium value. Since the initial energy $\Pi_{0}=\int_{\mathcal{B}_{c}} \Psi_{0} \mathrm{~d} V$ is zero, the internal energy of the model becomes negative during the phase transition. This follows Devonshire's approach [1,2] wherein the free energy $\Psi$ of $\mathrm{BaTiO}_{3}$ is zero in the cubic state.

The minimum possible value for $\Psi$ is given for the tetragonal crystal structure with homogeneous polarization $P_{0}$ in a state free of stress or electric field. Then, the model yields $\Psi_{0}=-0.5345 E_{0} P_{0}$, where $P_{0}=0.26$ $\mathrm{Cm}^{-2}$, is the remanent polarization of the relaxed domain and $E_{0}=2.182 \times 10^{7} \mathrm{Vm}^{-1}$ is the coercive electric field of the single domain (which is much greater than the coercive field of polydomain material). The results given in Sect. 4 are normalized using $\Psi_{0}, E_{0}$ and $P_{0}$.

The model simulates a region of material $\mathcal{B}_{c}$ within which electric displacement $\mathbf{D}$ is defined by

$$
\mathbf{D}=\mathbf{P}+\kappa_{0} \mathbf{E} \text { in } \mathcal{B}_{c}
$$


where $\kappa_{0}=8.854 \times 10^{-12} \mathrm{VmC}^{-1}$ is the permittivity of free space, and electric field $\mathbf{E}$ is derived from a scalar electric potential $\phi$ as

$$
\mathbf{E}=-\operatorname{Grad}[\phi] .
$$

The model fulfils Maxwell's electrostatic equations

$$
\begin{array}{ll}
\operatorname{Curl}[\mathbf{E}]=0 & \text { in } \mathcal{B}_{c}, \\
\operatorname{Div}[\mathbf{D}]=\rho & \text { in } \mathcal{B}_{c},
\end{array}
$$

where $\rho$ is the free charge density. Since the material is a ceramic insulator, we assume $\rho=0$. This, in combination with Eqs. 3 and 6, penalizes divergent polarization fields, such as 'head-to-head' or 'tail-to-tail' domains. At surfaces with outward normal direction $\mathbf{n}$ and surface charge density $q$, the electric displacement satisfies

$$
\mathbf{D} \cdot \mathbf{n}=q \text { on } \partial \mathcal{B}_{c}
$$

Equation (5) is automatically satisfied since $\operatorname{Curl}[\operatorname{Grad}[\phi]]=0$. Meanwhile, the mechanical part of the model assumes linear kinematics with displacements $\mathbf{u}$ and strain

$$
\varepsilon=\operatorname{symGrad}[\mathbf{u}] \text {. }
$$

Since we are not concerned with extrinsic forces, the balance of momentum reduces to

$$
\operatorname{Div}[\sigma]=0 \text { in } \mathcal{B}_{c}
$$

while at surfaces with traction $\mathbf{t}$,

$$
\mathbf{t}=\boldsymbol{\sigma} \cdot \mathbf{n} \text { on } \partial \mathcal{B}_{c} .
$$

Periodic boundary conditions on the pseudo-surface, $\partial \mathcal{B}_{c}$, of unit cells will be defined in Sect. 3, regulating the values of $\mathbf{P}, \phi$ and $\mathbf{u}$ on $\partial \mathcal{B}_{c}$.

The majority of simulations represent cubic volumes of material, modelled with up to $20^{3}=8000$ triquadratic finite elements of approximately $2 \mathrm{~nm}$ element size. This yields of the order of $10^{6}$ degrees of freedom. Extra degrees of freedom are introduced at domain walls, as they form, by enriching elements with quartic shape functions wherever the gradients of $\mathbf{P}$ exceed a critical magnitude; details of the enrichment method have been given by Muench and Krauß [44,45]. At the initial stage of the simulation, random values of $\mathbf{P}$ of magnitude $0.01 P_{0}$ are imposed on the system. This gives a perturbed near-zero polarization field corresponding to a state close to the maximum potential energy, representing the cubic state of the crystal. The subsequent evolution of domain patterns is due to the transformation from paraelectric to ferroelectric material at room temperature. Unless otherwise specified, the results show equilibrium states with $\dot{\mathbf{P}}=0$. Equilibrium was checked at the end of each simulation by increasing the time increment $\Delta t$ of the implicit time integration scheme by a factor of $10^{6}$ relative to the time steps used during the simulation, and checking for any change in polarization.

\section{Periodic boundary conditions}

Let us consider a nanoscale cubic unit cell $\mathcal{B}_{c}$ taken from the interior of a bulk single crystal with periodic domain pattern. Cartesian coordinates $x_{i}$ with orthogonal basis vectors $\mathbf{e}_{i}$ aligned to the edges of $\mathcal{B}_{c}$ are used. The origin $\mathbf{x}_{0}$ lies at the centre of $\mathcal{B}_{c}$, as shown in Fig. 1 . Dimensions are given by $L_{c}$ such that coordinates $x_{i} \in\left[-L_{c} / 2, L_{c} / 2\right], i=1 . .3$ and the volume $V_{c}=L_{c}^{3}$.

The surface $\partial \mathcal{B}_{c}$ of the unit cell has six faces with surface normal vectors

$$
\mathbf{n}^{I}=\mathbf{e}_{i} \quad \text { on } \mathcal{B}_{c}^{I}, I=i, \quad \mathbf{n}^{J}=-\mathbf{e}_{i} \quad \text { on } \mathcal{B}_{c}^{J}, J=i+3, i=1 . .3 .
$$

The centres of the faces $\partial \mathcal{B}_{c}^{I}, I=1 \ldots 6$, of $\mathcal{B}_{c}$ are defined by $\mathbf{o}^{I}=\mathbf{n}^{I} L_{c} / 2$. Position vectors on the faces $\partial \mathcal{B}_{c}^{I}$ are specified by $\mathbf{x}=\mathbf{o}^{I}+\mathbf{r}^{I}$ where

$$
\mathbf{r}^{1}=\mathbf{r}^{4}=\left(\begin{array}{c}
0 \\
x_{2} \\
x_{3}
\end{array}\right), \quad \mathbf{r}^{2}=\mathbf{r}^{5}=\left(\begin{array}{c}
x_{1} \\
0 \\
x_{3}
\end{array}\right), \quad \mathbf{r}^{3}=\mathbf{r}^{6}=\left(\begin{array}{c}
x_{1} \\
x_{2} \\
0
\end{array}\right)
$$

Each vector $\mathbf{r}^{I}$ inherits the parameters of the face $\partial \mathcal{B}_{c}^{I}$ as cartesian coordinates and is perpendicular to $\mathbf{n}^{I}$ as shown in Fig. 1b. 

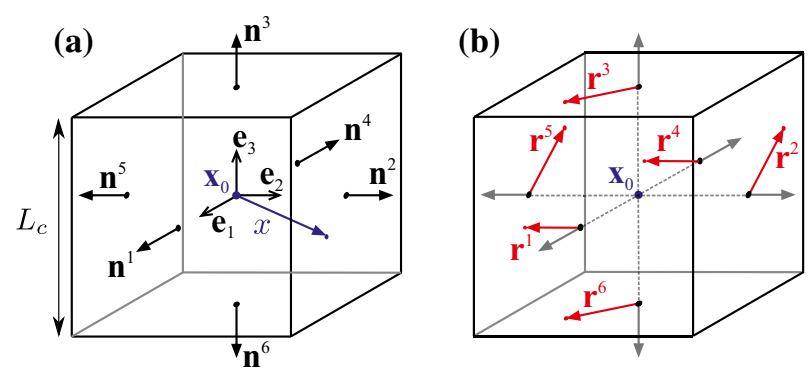

Fig. 1 a Cubic simulation volume $\mathcal{B}_{c}$ with cartesian coordinate system $\mathbf{e}_{i}$, centre $\mathbf{x}_{0}$, dimension $L_{c}$, and normal vectors $\mathbf{n}^{I}$ on faces $\partial \mathcal{B}_{c}^{I}$. b Tangential vectors $\mathbf{r}^{I}$ are shown on each surface $\partial \mathcal{B}_{c}^{I}$

\subsection{Standard periodic boundary condition}

A simple packing of unit cells is obtained by duplication and translation along $\mathbf{e}_{i}$ via cell length $L_{c}$. Using superscripts $I$ and $I+3$, the periodic boundary conditions on opposite faces $\partial \mathcal{B}_{c}^{I}$ and $\partial \mathcal{B}_{c}^{I+3}$ are then given by

$$
\begin{array}{ll}
\mathbf{P}\left(\mathbf{o}^{I}+\mathbf{r}^{I}\right)=\mathbf{P}\left(\mathbf{o}^{I+3}+\mathbf{r}^{I+3}\right), & I=1 . .3, \\
\phi\left(\mathbf{o}^{I}+\mathbf{r}^{I}\right)=\phi\left(\mathbf{o}^{I+3}+\mathbf{r}^{I+3}\right), & I=1 . .3, \\
\mathbf{u}\left(\mathbf{o}^{I}+\mathbf{r}^{I}\right)=\mathbf{u}\left(\mathbf{o}^{I+3}+\mathbf{r}^{I+3}\right), & I=1 . .3 .
\end{array}
$$

These periodic boundary conditions enforce zero average strain and electric field within the cell. In Sect. 3.3, we will introduce a side condition to allow for nonzero average strain. By then, Eq. (15) ensures that unit cells are geometrically compatible. Equation (13) also yields solenoidal polarization when averaged over $\mathcal{B}_{c}$ :

$$
\begin{aligned}
\int_{\mathcal{B}_{c}} \operatorname{Div}[\mathbf{P}] \mathrm{d} V & =\int_{\partial \mathcal{B}_{c}} \mathbf{P} \cdot \mathbf{n} \mathrm{d} A \\
& =\sum_{I=1}^{3} \int_{\partial \mathcal{B}_{c}^{I}} \mathbf{P}\left(\mathbf{o}^{I}+\mathbf{r}^{I}\right) \cdot \mathbf{n}^{I}+\mathbf{P}\left(\mathbf{o}^{I+3}+\mathbf{r}^{I+3}\right) \cdot \mathbf{n}^{I+3} \mathrm{~d} A=0 .
\end{aligned}
$$

Nevertheless, Eq. (16) does not imply that the polarization is locally divergence-free within $\mathcal{B}_{c}$.

\subsection{Reverse periodic boundary condition}

We next consider an assembly of cubes $\mathcal{B}_{c}$ to form an extended unit cell with internal symmetry. This can be achieved by translation along $\mathbf{e}_{i}$ combined with a $180^{\circ}$ rotation $\mathbf{R}^{I}$ about $\mathbf{e}_{i}$. The periodic conditions then read

$$
\begin{aligned}
& \mathbf{P}\left(\mathbf{o}^{I}+\mathbf{r}^{I}\right)=\mathbf{R}^{I} \mathbf{P}\left(\mathbf{o}^{I+3}+\mathbf{R}^{I} \mathbf{r}^{I+3}\right), \\
& \phi\left(\mathbf{o}^{I}+\mathbf{r}^{I}\right)=\phi\left(\mathbf{o}^{I+3}+\mathbf{R}^{I} \mathbf{r}^{I+3}\right), \\
& \mathbf{u}\left(\mathbf{o}^{I}+\mathbf{r}^{I}\right)=\mathbf{R}^{I} \mathbf{u}\left(\mathbf{o}^{I+3}+\mathbf{R}^{I} \mathbf{r}^{I+3}\right)
\end{aligned}
$$

The $180^{\circ}$ rotation tensors $\mathbf{R}^{I}$ are defined by

$$
\mathbf{R}^{I}=-11+2 \mathbf{n}^{I} \otimes \mathbf{n}^{I}, \quad \mathbf{R}^{I} \in \mathrm{SO}(3) \wedge \operatorname{Sym}(3),
$$

where 11 is the identity tensor and $\otimes$ yields the dyadic tensor product. With the restriction in Eq. (20), the electric polarization satisfies $q \equiv 0$ on $\partial \mathcal{B}_{c}$ when cubes $\mathcal{B}_{c}$ are assembled after duplication, translation along $\mathbf{e}_{i}$ via cell length $L_{c}$, and rotation by $\mathbf{R}^{I}$. This reverse periodic boundary condition can be applied to one, two, or three faces simultaneously. In the present work, the reverse condition is applied on a single pair of opposite faces, with standard periodic boundary conditions employed on the other faces.

As with the standard periodic boundary condition, the reverse periodic boundary condition yields solenoidal polarization averaged over $\mathcal{B}_{c}$. Similarly, this boundary condition implies that the mean strain and electric field vanish over the extended unit cell. Thus, the assembly of cells $\mathcal{B}_{c}$ with reverse periodic boundary condition 

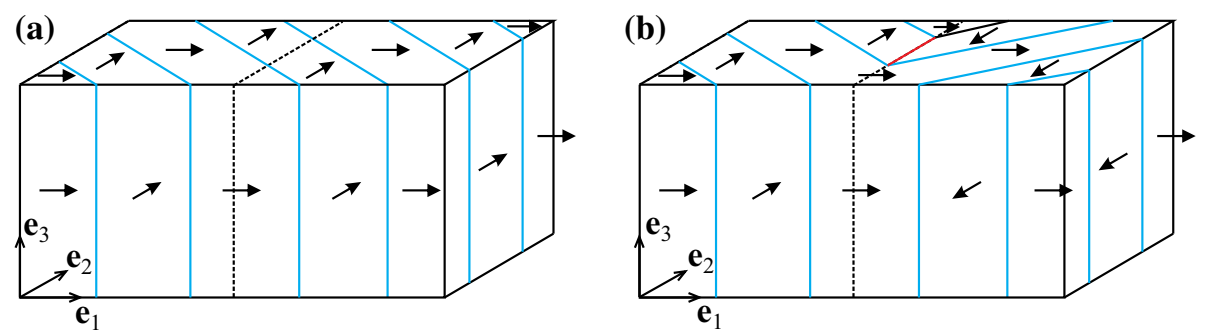

Fig. 2 Translation of cubes $\mathcal{B}_{c}$ in the $\mathbf{e}_{1}$-direction; polarization is indicated by arrows. a An assembly of two cubes using the standard periodic boundary condition to yield a rank-1 laminate. $\mathbf{b}$ An assembly of two cubes using the reverse boundary condition with rotation around $\mathbf{e}_{1}$ and translation; this produces a rank-2 laminate with a $180^{\circ}$ domain wall, highlighted in red . (Color figure online)

does not require any macroscopic electric field to stabilize it. Figure 2 illustrates the effect of the standard and reverse boundary conditions. The reverse periodic boundary condition encourages the formation of additional $180^{\circ}$ domain walls and hence more complex domain patterns.

In summary, the reverse periodic boundary condition is applied to accelerate the search for complex domain patterns at smaller RVE's. It enforces a double cell symmetry condition, without increasing the computational volume. This boundary condition encourages periodic patterns with oppositely polarized domains to form, in order to maintain an overall electric field neutrality.

\subsection{Side conditions for strained states}

Next, we introduce mechanical side conditions to apply four different representative strained states. We use these representative strained states to explore the general trends in temporal evolution of polarization patterns and to explore the complexities of 3D-domain arrangements.

When $\mathrm{BaTiO}_{3}$ transforms freely from the cubic phase to the tetragonal phase, the transformation results in a remanent strain $\boldsymbol{\varepsilon}_{r}$, which is non-isochoric. Three variants of $\boldsymbol{\varepsilon}_{r}$ are observed, with the tetragonal $c$ axis aligned to $\mathbf{e}_{1}, \mathbf{e}_{2}$ and $\mathbf{e}_{3}$, respectively. The cubic lattice constant $a_{c u b}$ is replaced by tetragonal lattice constants $c_{t e t}$ and $a_{t e t}$, respectively, giving remanent strain components

$$
\varepsilon_{c}=\frac{c_{t e t}-a_{c u b}}{a_{c u b}}>0, \quad \varepsilon_{a}=\frac{a_{t e t}-a_{c u b}}{a_{c u b}}<0 .
$$

Thus, the three strain variants are

$$
\boldsymbol{\varepsilon}_{1}^{0}=\left(\begin{array}{ccc}
\varepsilon_{c} & 0 & 0 \\
0 & \varepsilon_{a} & 0 \\
0 & 0 & \varepsilon_{a}
\end{array}\right), \quad \boldsymbol{\varepsilon}_{2}^{0}=\left(\begin{array}{ccc}
\varepsilon_{a} & 0 & 0 \\
0 & \varepsilon_{c} & 0 \\
0 & 0 & \varepsilon_{a}
\end{array}\right), \quad \boldsymbol{\varepsilon}_{3}^{0}=\left(\begin{array}{ccc}
\varepsilon_{a} & 0 & 0 \\
0 & \varepsilon_{a} & 0 \\
0 & 0 & \varepsilon_{c}
\end{array}\right) .
$$

In each case, the $c$ axis aligns with the polarization $\mathbf{P}$ in the minimum energy state. For $\mathrm{BaTiO}_{3}$, set $a_{c u b}=$ $4.000 \AA, c_{t e t}=4,0328 \AA$, and $a_{t e t}=3.9892 \AA$, based on experimental data and theoretical results presented in [48]. Then, $\varepsilon_{c}=0.0082$ and $\varepsilon_{a}=-0.0027$ giving $2 \varepsilon_{a}+\varepsilon_{c}=0.0028$, which indicates that the unit cell undergoes a non-isochoric process if unconstrained. Thus, it is desirable for the simulations to allow an overall straining of $\mathcal{B}_{c}$, corresponding to displacement of the boundaries $\partial \mathcal{B}_{c}$.

The strain-free boundary conditions of Eqs. (15) and (19) would suppress an average remanent strain, resulting in a stressed state. To enable a state closer to stress-free conditions, we introduce scalar parameters $\lambda_{i}, \mathrm{i}=1 . .3$, tracking approximate volume fractions of each of the three strain variants, as indicated by the local polarization direction:

$$
\lambda_{i}:=\frac{1}{V_{c}} \int_{\mathcal{B}_{c}} \frac{\left|\mathbf{P} \cdot \mathbf{e}_{i}\right|}{P_{0}} \mathrm{~d} V, \quad i=1 . .3 .
$$

Using the volume fractions $\lambda_{i}$ and the remanent strain variants from Eq. (22), define an overall remanent strain

$$
\overline{\boldsymbol{\varepsilon}}_{r}:=\frac{1}{V_{c}} \int_{\mathcal{B}_{c}} \sum_{i=1}^{3} \frac{\left|P_{i}\right|}{P_{0}} \boldsymbol{\varepsilon}_{i}^{0} \mathrm{~d} V=\sum_{i=1}^{3} \boldsymbol{\varepsilon}_{i}^{0} \frac{1}{V_{c}} \int_{\mathcal{B}_{c}} \frac{\left|\mathbf{P} \cdot \mathbf{e}_{i}\right|}{P_{0}} \mathrm{~d} V=\sum_{i=1}^{3} \lambda_{i} \boldsymbol{\varepsilon}_{i}^{0}, \quad \overline{\boldsymbol{\varepsilon}}_{r} \in \text { Diag. }
$$


Note that $\overline{\boldsymbol{\varepsilon}}_{r}$ is not the actual strain or strain average of the simulation, but instead is a notional remanent strain that is consistent with the current polarization state of the simulation. Since it results from an average over $\mathcal{B}_{c}$, $\overline{\boldsymbol{\varepsilon}}_{r}$ is not a function of position. The overall volume change due to average remanent strain $\overline{\boldsymbol{\varepsilon}}_{r}$ is

$$
\Delta V=V_{c} \operatorname{tr}\left[\overline{\boldsymbol{\varepsilon}}_{r}\right]=V_{c}\left(\lambda_{1}+\lambda_{2}+\lambda_{3}\right)\left(2 \varepsilon_{a}+\varepsilon_{c}\right) .
$$

Now consider imposing some state of average strain of the form $\bar{\varepsilon} \in$ Diag. This could be achieved by replacing the displacement boundary conditions, Eqs. (15) and (19), with

$$
\mathbf{u}\left(\mathbf{o}^{I}+\mathbf{r}^{I}\right)=\mathbf{u}\left(\mathbf{o}^{I+3}+\mathbf{r}^{I+3}\right)+\overline{\boldsymbol{\varepsilon}} \cdot \mathbf{n}^{I} L_{c}, \quad I=1 . .3,
$$

for the standard periodic boundary condition, and

$$
\mathbf{u}\left(\mathbf{o}^{I}+\mathbf{r}^{I}\right)=\mathbf{R}^{I} \mathbf{u}\left(\mathbf{o}^{I+3}+\mathbf{R}^{I} \mathbf{r}^{I+3}\right)+\overline{\boldsymbol{\varepsilon}} \cdot \mathbf{n}_{I} L_{c}, \quad I=1 . .3 .
$$

for the reverse periodic boundary condition. Equivalently, we may keep Eqs. (15) and (19), and replace (8) by the side condition

$$
\boldsymbol{\varepsilon}=\operatorname{symGrad}[\mathbf{u}]+\overline{\boldsymbol{\varepsilon}},
$$

taking care to adjust the definition of Helmholtz free energy in Eq. (2) accordingly. This latter approach is used in the present work. In order to control the average strain during transformation from cubic to tetragonal in differing ways, the three diagonal elements of $\overline{\boldsymbol{\varepsilon}}$ can be defined using parameters $f_{1}, f_{2}$, and $f_{3}$ by analogy with Eq. (24), such that

$$
\overline{\boldsymbol{\varepsilon}}=\sum_{i=1}^{3} f_{i} \boldsymbol{\varepsilon}_{i}^{0}, \quad \overline{\boldsymbol{\varepsilon}} \in \text { Diag. }
$$

The parametrization with $f_{i}$ aids the formulation of different types of constraints or strain controls. For example, setting $f_{1}=1$ and $f_{2}=f_{3}=0$ recovers the strain state of a stress-free single domain with $c$ axis aligned to $\mathbf{e}_{1}$. By contrast, setting all $f_{i}=0, i=1 \ldots 3$, produces a state free of average strain, while setting all $f_{i}=1 / 3$ produces an equiaxed volume dilation that matches the dilation of the cubic-tetragonal phase transformation.

Simulations were conducted with four specific side conditions, as follows: First, the average strain-free case, where

$$
f_{1}=f_{2}=f_{3}=0,
$$

is denoted Isochoric Ferroelectric Phase Transition (IFPT). Thus, the volume of $\mathcal{B}_{c}$ is held constant during the simulation. A negative spherical stress is likely to arise to restrain $\overline{\boldsymbol{\varepsilon}}_{r}$ if the material evolves polarized domains such that volume fractions $\lambda_{i}>0$.

By contrast, a low stress phase transition may be promoted by

$$
f_{i}=\lambda_{i}, \quad i=1 . .3 .
$$

Here, $\overline{\boldsymbol{\varepsilon}}$ adapts continuously to match $\overline{\boldsymbol{\varepsilon}}_{r}$; this condition will be denoted Adaptive Spherical Stress Free (ASSF). Note that ASSF may generally yield unequal strains along the three axes, but tracks any volume dilation accompanying the transformation, so that typically $\operatorname{tr}[\overline{\boldsymbol{\varepsilon}}]=\left(2 \varepsilon_{a}+\varepsilon_{c}\right)=0.0028$ at equilibrium.

A third condition enforces equal average straining along each axis while matching the expected volume dilation due to transformation:

$$
f_{1}=f_{2}=f_{3}=1 / 3 .
$$

This will be denoted as the Isotropic Spherical Stress Free (ISSF) case. This side condition tends to eliminate any average spherical stress.

Finally, a common arrangement for materials bound by a substrate, or subjected to a uniaxial mechanical loading, is that the strain state retains two equal principal strains. Combining this with the restriction to match the volumetric dilation due to the phase transformation gives:

$$
f_{1}=a, f_{2}=f_{3}=b, \quad a+2 b=1, \quad a \neq 1 / 3 .
$$




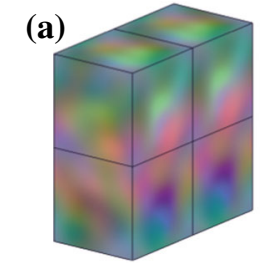

(c)

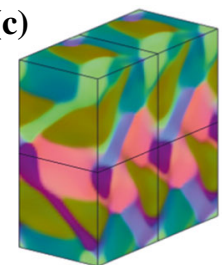

(e)

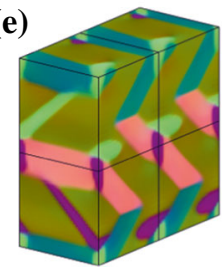

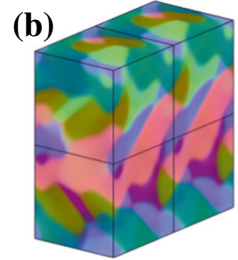

(d)

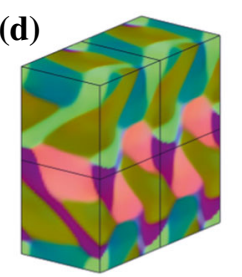

(f)

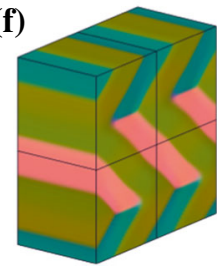

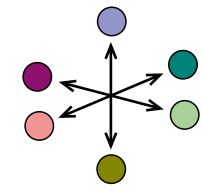

(g)

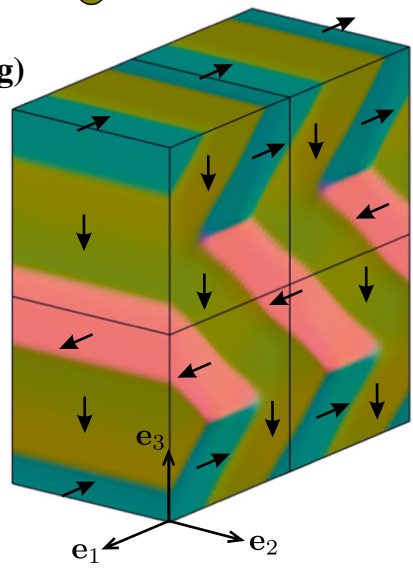

Fig. 3 3D simulation results for a cubic region, with the reverse periodic boundary condition applied in $\mathbf{e}_{3}$ direction. a-f Successive stages in the simulation before equilibrium are reached. $\mathbf{g}$ Equilibrium state, showing polarization directions with arrows

The particular case when $b=1 / 2$ we denote Transversely Isotropic Deformation (TID). Note that all four cases allow for locally inhomogeneous strain within $\mathcal{B}_{c}$. Further, the volume fractions $\lambda_{i}$ need not generally coincide with imposed strain parameters $f_{i}$, except for the case of ASSF, where there is continuous tracking to match these parameters.

The strained states might bear similarities to experimentally imposed mechanical strains. However, the spirit of the current work is to indicate the general trends for complexity and types of patterns that form in bulk ferroelectrics.

\section{Results and discussion}

Domain topologies resulting from the use of periodic boundary conditions in 3-dimensions, combined with each of the four strain conditions (IFPT, ASSF, ISSF, TID), are presented in this section. It is instructive first to consider the evolution of domains within a single simulation, then to explore the effect of the periodic cell size in the range $9-41 \mathrm{~nm}$ on the topologies that may form. Finally, a cell size large enough to generate complex domain patterns $(41 \mathrm{~nm})$ is selected and the domain topologies resulting from each set of boundary conditions are described.

\subsection{Evolution of a 2-dimensional domain topology}

In this example, a cube $\mathcal{B}_{c}$ of size $L_{c}=33 \mathrm{~nm}$ is simulated using $16 \times 16 \times 16$ elements. Standard periodic boundary conditions are used on the opposite face pairs $\left(\partial \mathcal{B}_{c}^{1}, \partial \mathcal{B}_{c}^{4}\right)$ and $\left(\partial \mathcal{B}_{c}^{2}, \partial \mathcal{B}_{c}^{5}\right)$. Meanwhile, the reverse periodic boundary condition is applied to the face pair $\left(\partial \mathcal{B}_{c}^{3}, \partial \mathcal{B}_{c}^{6}\right)$. The adaptive spherical stress-free condition (ASSF) on average strain is applied.

Figure 3 shows a series of states of the simulation as it evolves towards equilibrium; each image shows four copies of the simulated volume $\mathcal{B}_{c}$ to illustrate the periodic continuation in the $\mathbf{e}_{1}$ and $\mathbf{e}_{3}$ directions. The reverse periodic boundary condition encourages (but does not enforce) the formation of a $180^{\circ}$ domain wall normal to the $\mathbf{e}_{3}$ direction.

In the early stages of the simulation, the local values of $|\mathbf{P}|$ are much less than $P_{0}$, but the energetic driving force for the formation of polarized regions rapidly produces domains. These initially nucleate in a random, 
high energy pattern, and gradually resolve sharply defined $90^{\circ}$ and $180^{\circ}$ domain walls. As can be seen from Fig. $3 \mathrm{c}-\mathrm{e}$, the domain pattern is complex as it evolves towards equilibrium, but eventually reaches a simpler, lower energy state.

The final equilibrium state shows a herringbone-type pattern that was also identified by Tsou et al. [5], using a sharp interface model, as a member of the family $\{1314\}$ of compatible rank-2 domain patterns. In this case, the final pattern is essentially 2-dimensional, with the polarization vector always lying in the $\mathbf{e}_{1}-\mathbf{e}_{3}$ plane; this was not forced, but rather was a natural outcome of the relaxation towards a minimum energy state. The same pattern was noted in earlier work using a phase field model, but in a 2-dimensional simulation with a 40nm periodic cell size [14]. However, in that study a fixed average strain was imposed to stabilize the pattern. Here, the adaptive strain condition allows the topology to form and adopt an average strain state that minimizes energy.

\subsection{Cell size variation with standard periodic boundary conditions}

In this section, the effect of periodic cell size by varying the cell size $L_{c}$ from 9 to $41 \mathrm{~nm}$ is under investigation. Below about $9 \mathrm{~nm}$ size, the cell is too small to support multiple domains (domain wall widths in the model are in the region of $2-3 \mathrm{~nm}$. Beyond $41 \mathrm{~nm}$ the computation becomes excessively cumbersome due to the scaling of the number of degrees of freedom in the problem. From our previous work on scale effects in polarization patterns [13], we expect that increasing the cell size will enable polarization patterns with multiple domain walls to form. However, in the current work we stop at $41 \mathrm{~nm}$ cell size because it is sufficient for complex domain patterns to form within practical computation time.

For the smaller cell sizes $(9-25 \mathrm{~nm})$, elements of size $1 \mathrm{~nm}$ were employed. Cell sizes of 33 and $41 \mathrm{~nm}$ were simulated with $16^{3}$ and $20^{3}$ elements, respectively. The standard periodic boundary condition is used as a representative boundary condition to explore the effect of cell size variation. We expect a similar trend in the results with reverse periodic boundary conditions. Several runs of the model were performed with different random initial polarization perturbations; these runs can reach different equilibrium states. The results in Fig. 4 show the final domain topologies with lowest overall Helmholtz free energy. Additionally, the side condition defining the average strain was varied to explore each of the four cases defined in Sect. 3.3.

As shown in Fig. 4, simple $90^{\circ}$ domain stripes arise in most of the simulations. However, the TID constraint, which imposes a strain condition close to the free strain of the single domain, strongly encourages a monodomain to form. Generally, smaller cell sizes result in simpler domain patterns. Only the $41 \mathrm{~nm}$ cell is large enough that more complex domain patterns can form if this reduces the energy. Thus, for the subsequent study of domain topologies, the $41 \mathrm{~nm}$ cell size is employed.

Next, let us define volume fractions $\alpha_{i}, \beta_{i}, i=1,2,3$, to assist in identifying the presence of $90^{\circ}$ and $180^{\circ}$ domains:

$$
\alpha_{i}=\left|\int_{V_{c}} \frac{P_{i}}{P_{0}} \frac{\mathrm{d} V}{V_{p}}\right|, \quad \beta_{i}=\int_{V_{c}} \frac{\left|P_{i}\right|}{P_{0}} \frac{\mathrm{d} V}{V_{p}}, \quad V_{p}=\sum_{i=1}^{3} \int_{V_{c}} \frac{\left|P_{i}\right|}{P_{0}} \mathrm{~d} V .
$$

where the integrals are defined over the entire periodic cell volume $V_{c}$ (note that this may contain multiple, transformed copies of cube $\mathcal{B}_{c}$ ). In Eq. (34), $V_{p}$ gives the volume fraction of the periodic cell that is polarized into domains. At equilibrium this is normally close to $100 \%$. The values of $\alpha_{i}$ and $\beta_{i}$ give, respectively, the fractional net polarization along each axis and the fraction of domains aligned with each axis, normalized in each case by the total volume fraction of domains. For convenience, we sort these volume fractions such that $\alpha_{I} \geq \alpha_{I I} \geq \alpha_{I I I}$ and $\beta_{I} \geq \beta_{I I} \geq \beta_{I I I}$, expressing each value as a percentage.

To understand the meaning of $\boldsymbol{\alpha}$ and $\boldsymbol{\beta}$ in terms of domain topologies, note that for a simple monodomain $\boldsymbol{\alpha}=$ $\boldsymbol{\beta}=[100,0,0]$. Alternating $180^{\circ}$ domains with equal volume fractions give $\boldsymbol{\alpha}=[0,0,0], \boldsymbol{\beta}=[100,0,0]$, while alternating $90^{\circ}$ domains with equal volume fractions give $\boldsymbol{\alpha}=[50,50,0], \boldsymbol{\beta}=[50,50,0]$. Differences between $\boldsymbol{\alpha}$ and $\boldsymbol{\beta}$ indicate the formation of oppositely polarized regions. Values in $\boldsymbol{\alpha}$ close to zero indicate that there are directions with little or no net polarization while a value in $\boldsymbol{\beta}$ close to zero would indicate the presence of an axis to which few or no domains have their $c$ axis aligned. Both measures are invariant to rotations or reflections of the periodic unit cell in the symmetry group of the cube. Table 1 shows the normalized Helmholtz free energy along with $\boldsymbol{\alpha}$ and $\boldsymbol{\beta}$ as percentage values for the equilibrium states, computed with $41 \mathrm{~nm}$ cubes.

The normalized overall free Helmholtz energy $\bar{\Psi}=\int_{\mathcal{B}_{c}} \Psi / L_{c}^{3} \mathrm{~d} V$ is always negative, as the zero datum is the high energy, cubic, state. However, the ratio $\bar{\Psi} / \Psi_{0}$, where $\Psi_{0}=-0.5345 E_{0} P_{0}$ corresponds to the energy 
$L_{c}[n m]$

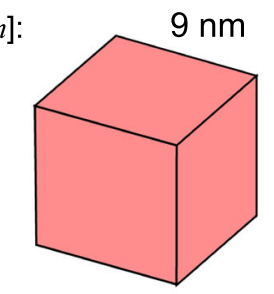

IFPT
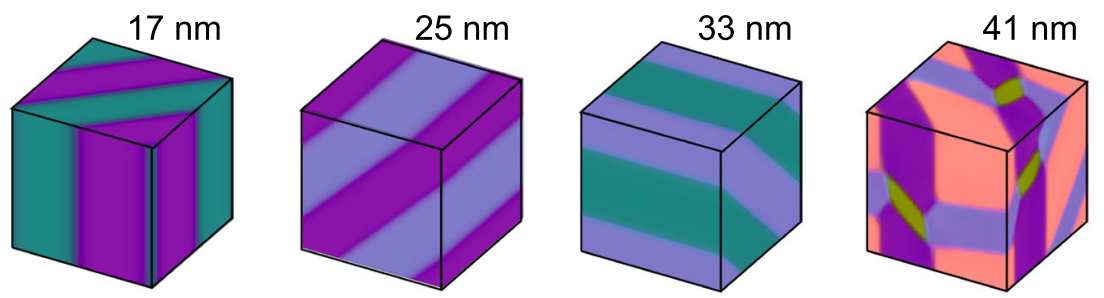

ISSF
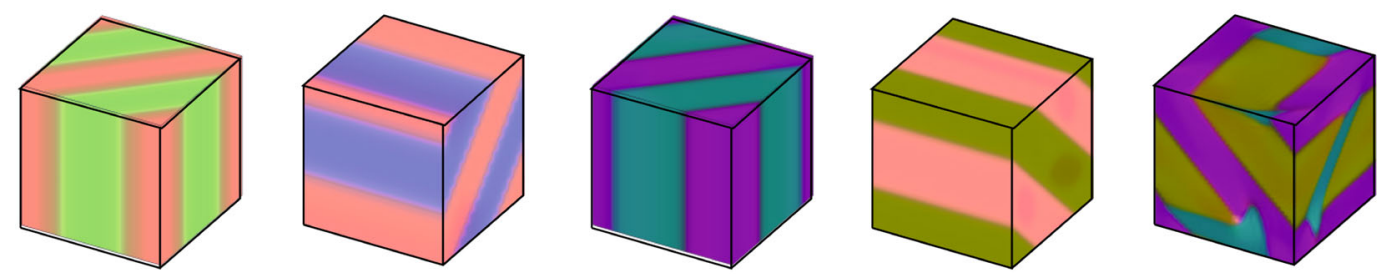

TID
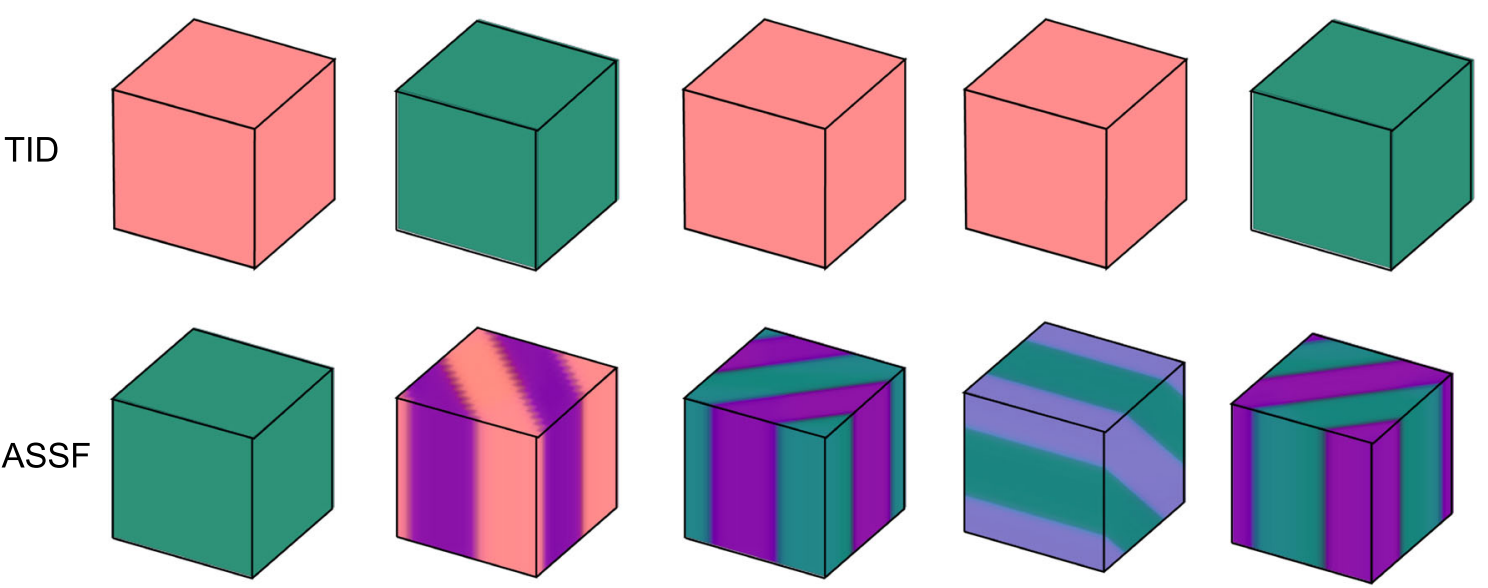

Fig. 4 Equilibrium domain topologies with standard periodic boundary conditions, with varying strain conditions and cell dimensions in the range from $9-41 \mathrm{~nm}$

of a stress-free single domain, is a positive number less than 1, see Table 1. Imposing a strained state (IFPT, ISSF, TID) often does not lead to the single domain solution as can be seen from the $\boldsymbol{\alpha}, \boldsymbol{\beta}$ values; even when a monodomain does result, the energy is higher than that of the stress-free state. Allowing the periodic cell to adapt its strain as domains develop (ASSF) results in the lowest energy solutions, which may be a simple monodomain, but can develop a domain pattern if the random starting conditions nucleate one. The isochoric conditions (IFPT) result in the highest vales of free energy, as the periodic cell is rigidly constrained to match that of the cubic state.

\subsection{Domain patterns simulated with standard periodic boundary conditions}

From now on, we only consider results from simulations of $41 \mathrm{~nm}$ cells. Figure 5 shows the domain pattern for the isochoric ferroelectric phase transition (IFPT) and using the standard periodic boundary conditions. Since these conditions strongly constrain the formation of domains aligned with any one preferred direction, it is expected that a fully 3-dimensional pattern of domains with some regions polarized parallel to each of the three directions $\mathbf{n}^{I}(I=1 \ldots 3)$ is likely to form. Tsou et al. [5] classified the simplest compatible laminate of domains that has all three tetragonal strain variants present as a rank-2 laminate in a family $\{1325\}$ that has a mixture of $90^{\circ}$ and $180^{\circ}$ domains. This pattern is also the outcome of the phase field simulation: Fig. 5b-d shows cross sections of the pattern that identify the polarized directions of the domains.

Close examination reveals that the laminate does not have perfectly planar interfaces between domains, but instead the intersections of domains form complicated angular shapes that result in vortex-like regions at the triple junctions where domains polarized along the $\pm \mathbf{e}_{3}$ and $-\mathbf{e}_{2}$ directions meet. There has been great interest 
Table 1 Helmholtz free energy and volume fractions $(\boldsymbol{\alpha}, \boldsymbol{\beta})$ for standard and reverse periodic boundary conditions $(\mathrm{PBC})$ and strain controls in the $L_{c}=41 \mathrm{~nm}$ cubes. The parameters $f_{i}$ define the imposed strain controls and $\lambda_{i}$ tracks volume fractions of domains in the evolving simulation for the adapted spherical stress-free case (ASSF)

\begin{tabular}{llll}
\hline & & Standard PBC & Reverse PBC \\
\hline IFPT & $\bar{\Psi} / \Psi_{0}$ & 0.520 & 0.472 \\
$f_{i}=0$ & $\boldsymbol{\alpha}$ & $39,30,14$ & $36,0,0$ \\
$\mathrm{ISSF}$ & $\overline{\boldsymbol{\beta}} / \Psi_{0}$ & $42,31,27$ & $39,33,28$ \\
$f_{i}=1 / 3$ & $\boldsymbol{\alpha}$ & 0.642 & 0.588 \\
$\mathrm{TID}$ & $\boldsymbol{\beta}$ & $40,39,11$ & $37,0,0$ \\
$f_{1}=1 / 2$ & $\Psi / \Psi_{0}$ & $44,40,16$ & $40,31,29$ \\
$f_{2}=f_{3}=1 / 4$ & $\boldsymbol{\alpha}$ & 0.602 & 0.565 \\
ASSF & $\boldsymbol{\beta}$ & $100,0,0$ & $0,0,0$ \\
$f_{i}=\lambda_{i}$ & $\bar{\Psi} / \Psi_{0}$ & $100,0,0$ & $100,0,0$ \\
& $\boldsymbol{\alpha}$ & 0.870 & 0.752 \\
\hline
\end{tabular}
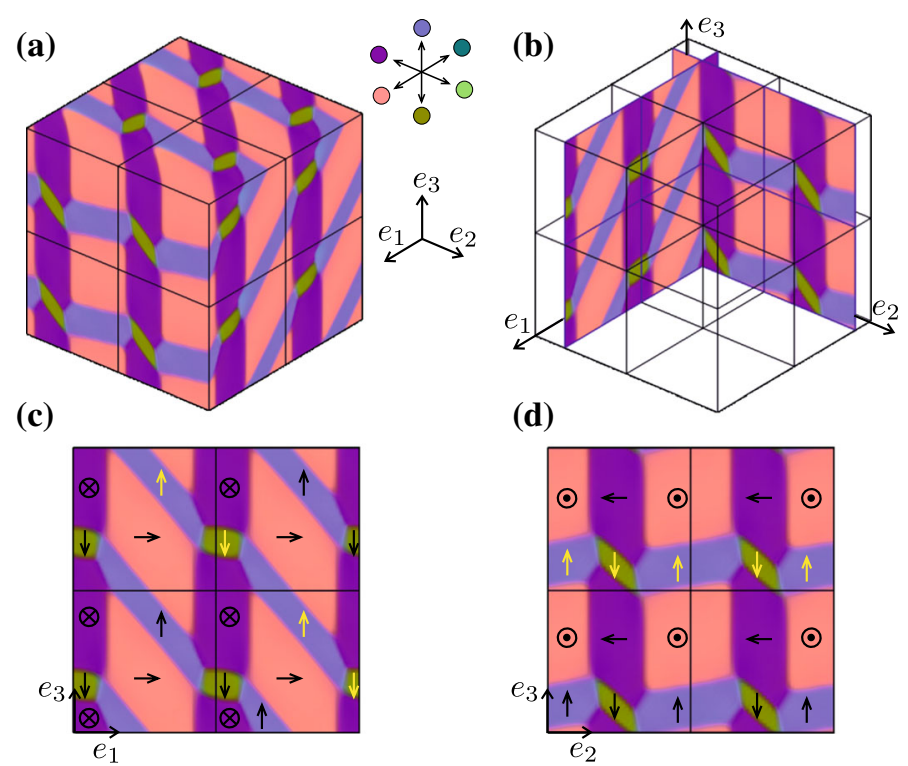

Fig. 5 Equilibrium domain pattern computed using standard periodic boundary conditions and the isochoric mechanical side condition (IFPT) a 3D assembly of cells. b-d Cross sections through the pattern. Sequences of $180^{\circ}$ domain walls are highlighted by yellow arrows. (Color figure online)

in such vortex centres $[11,38,49]$ because of their potential use in applications such as memory storage, and their interesting topological properties as skyrmions. The simulations here suggest that it may be possible to generate arrays of vortices by suitably constraining crystals during the cubic-tetragonal phase transition.

Changing the mechanical side condition to the isotropic spherical stress-free case (ISSF) keeps the average strain spherical (equal in all directions) but reduces the energy by allowing volumetric expansion to match the phase transformation, see Table 1. The result shown in Fig. 6 is once again a rank-2 domain pattern that was identified in the study of Tsou et al. [5]. In contrast to the IFPT solution in Fig. 5, it does not contain $180^{\circ}$ domain walls. However, this pattern, in the family named $\{1315\}$, contains an electrically incompatible boundary where domains polarized in the $-\mathbf{e}_{1}$ direction meet domains polarized in the $-\mathbf{e}_{2}$ direction. As can be seen in Fig. 6, this frustrated boundary adopts a curved shape to reduce energy. Thus, the equilibrium state is once again an imperfect laminate that would not be expected from consideration of the constrained, sharp interface theory alone. We note that domain arrangements observed on the cross sections of the multi-cell assembly in Figs. 5 and 6, bear resemblances to polarization patterns in thin films, simulated using phase field methods [51]. For example, the vortex-like regions at triple junctions and the curved domain walls are predicted to form on the free surface of a ferroelectric thin-film geometry. 

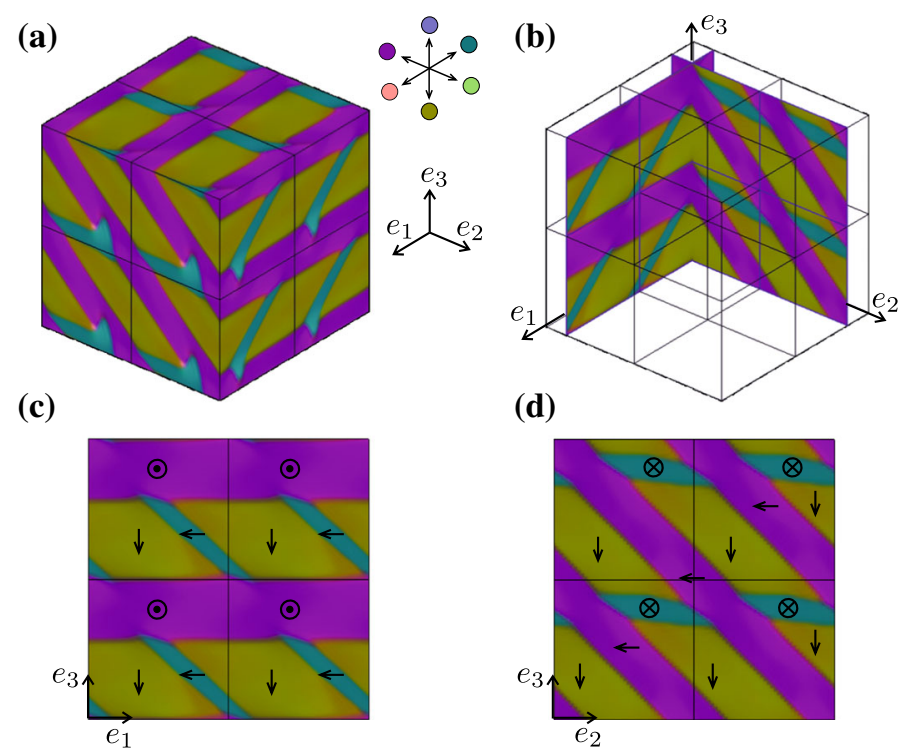

Fig. 6 Equilibrium domain pattern computed using standard periodic boundary conditions and the isotropic spherical stress-free constraint (ISSF). a 3D assembly of cells. b-d Cross sections through the pattern. No sequences of $180^{\circ}$ domain walls can be found

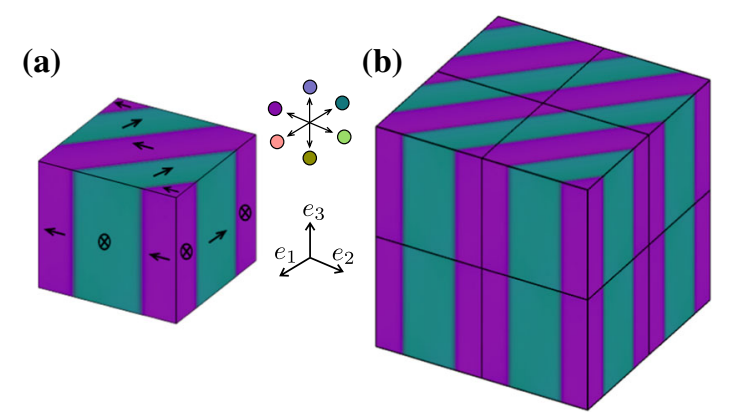

Fig. 7 Equilibrium domain pattern computed using standard periodic boundary conditions and the adapted spherical stress-free strain condition (ASSF). a Polarization in the simulation volume. b An assembly of periodic cells

The transversely isotropic deformation condition (TID) enforces a strain close to that of the unstressed single domain, and the simulation then produces a monodomain equilibrium state as shown in Fig. 4. However, the adapted spherical stress-free strain condition (ASSF), which continuously adapts the strain to be consistent with the average polarization, allows other patterns to form. The monodomain is a possible solution, but in our simulations the ASSF condition more commonly resulted in periodic $90^{\circ}$ domains, as shown in Fig. 7. The TID and ISSF cases have lower energy than that of IFPT, but the adaptive case (ASSF) gives the lowest energy of all, since it relaxes the average strained state as far as possible while retaining periodicity.

In summary, the model tends to form more complex domain patterns, including some of the vortex structures and ribbon-like domains shown in Figs. 5 and 6, if the mechanical side condition of periodic boundary conditions constrains the ferroelectric phase transition. The reason for this is that the constraint forces the material to produce a mixture of domains to match the imposed strain. In experimental work, the constraint can be imposed by the use of epitaxial growth with lattice mismatch. This method has been used in strontium titanate, for example, to provide biaxial tensile stresses that induce incipient ferroelectricity [52]. By contrast, our work suggests that lattice mismatch or stress gradients in the sample could be used in thin films of ordinary ferroelectrics to promote complex domain structures, or to encourage specific patterning of domains [53]. For example, a stress gradient generated by an atomic force microscopy tip was used to mechanically write polarization patterns on a barium titanate thin film [54]. 
(a)

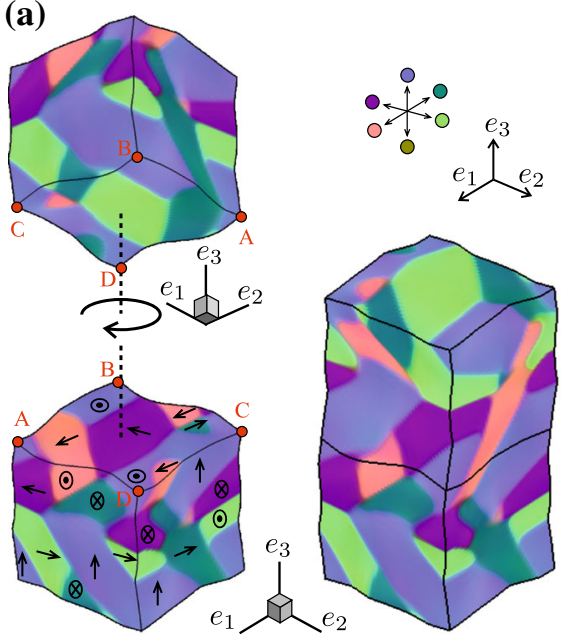

(b)

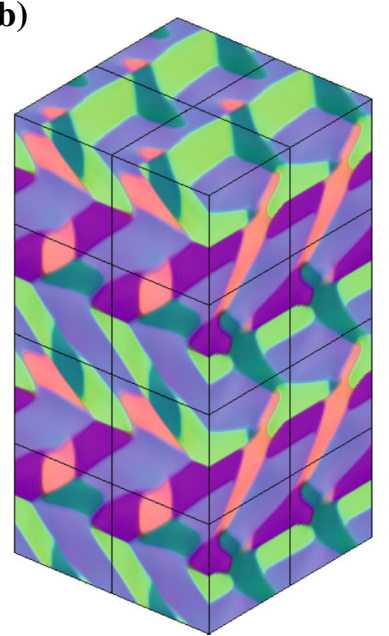

Fig. 8 Equilibrium domain pattern computed with the reverse periodic boundary condition along the $\mathbf{e}_{3}$ direction and the isochoric side condition (IFPT). a Showing the deformation exaggerated, and the method of assembly of simulated volumes $\mathcal{B}_{c}$ to produce the double volume periodic cell. b An assembly of periodic cells

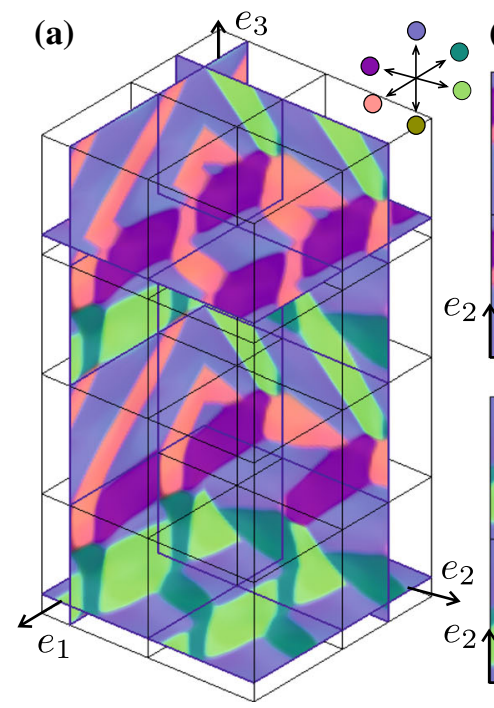

(b)

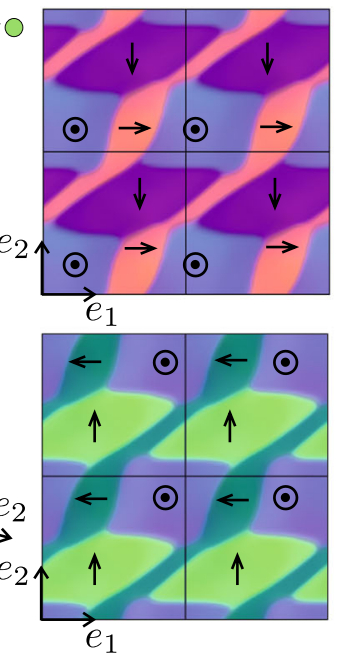

(c)

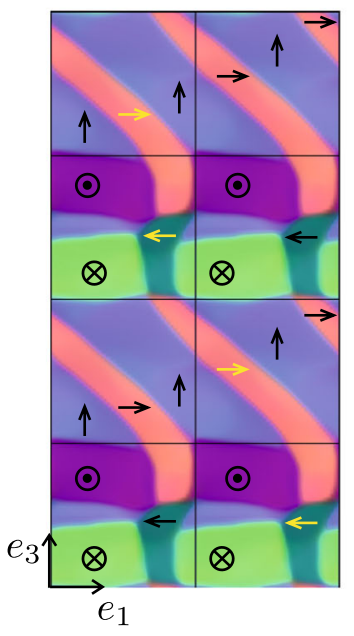

(d)

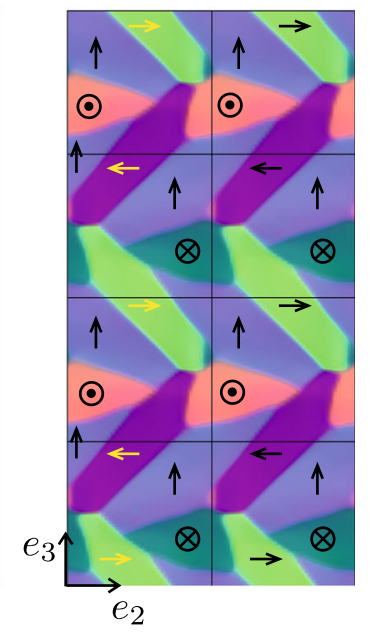

Fig. 9 Cross sections through the multi-cell assembly for IFPT and reverse periodic boundary condition along the $\mathbf{e}_{3}$ direction. a Showing the locations of the slices. b-d Cross sections normal to: $\mathbf{b}$ the $\mathbf{e}_{3}$ direction, $\mathbf{c}$ the $\mathbf{e}_{2}$ direction, $\mathbf{d}$ the $\mathbf{e}_{1}$ direction

\subsection{Domain patterns simulated with the reverse periodic boundary condition}

We observe two consequences of the reverse periodic boundary condition along the $\mathbf{e}_{3}$ axis: it doubles the periodic cell size and enforces symmetry such that the average polarization in perpendicular directions (here it is $\mathbf{e}_{1}$ and $\mathbf{e}_{2}$ ) is zero. Both effects have their origin in the enhanced continuation rule, where translation and rotation by $180^{\circ}$ along the $\mathbf{e}_{3}$ axis is superimposed. This encourages the formation of $180^{\circ}$ domain walls normal to the $\mathbf{e}_{3}$ direction within the RVE, as already shown in Fig. 3 for the $33 \mathrm{~nm}$ cell. Now, we present results for $41 \mathrm{~nm}$ sized cells and start with the isochoric side condition (IFPT) in Figs. 8, 9. The solution is a complex domain pattern containing $90^{\circ}$ and $180^{\circ}$ domain walls, some of which are curved, and including five of the six possible polarization variants (only the $-\mathbf{e}_{3}$ direction has no domains).

We show exaggerated deformations in Fig. 8a to illustrate that, although the volumetric strain is held at zero, there is nevertheless local straining of the periodic cell. The pattern has roughly equal volumes of domains with their $c$ axis aligned parallel to the $\mathbf{e}_{1}, \mathbf{e}_{2}$ and $\mathbf{e}_{3}$ directions, as indicated by $\boldsymbol{\beta}=[39,33,28]$. Vortex centres are evident at the junctions of the $\mathbf{e}_{3}$ and $\pm \mathbf{e}_{2}$ domains in Fig. 9d. 


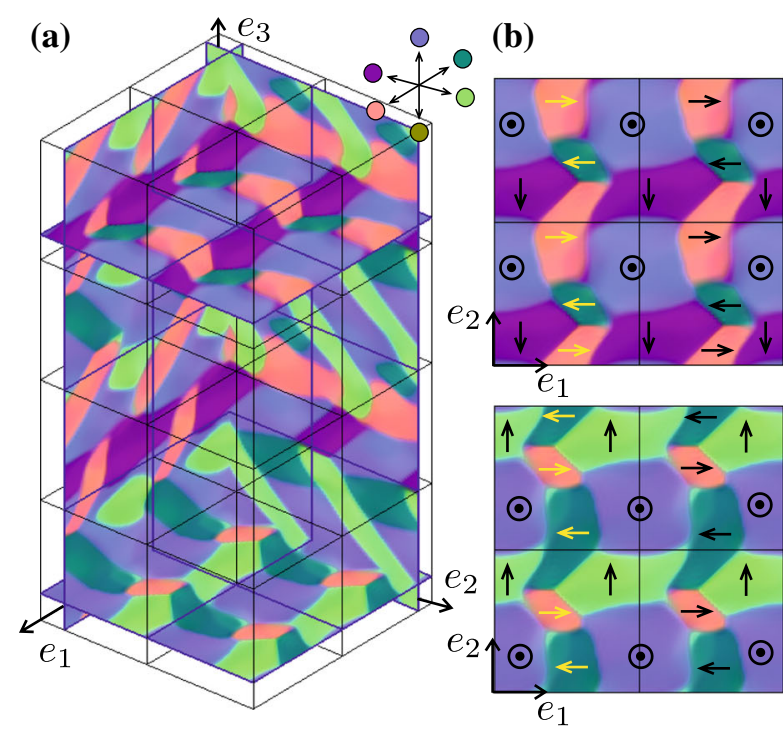

(c)

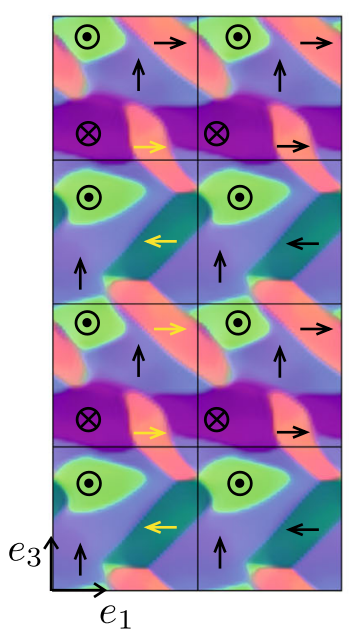

(d)

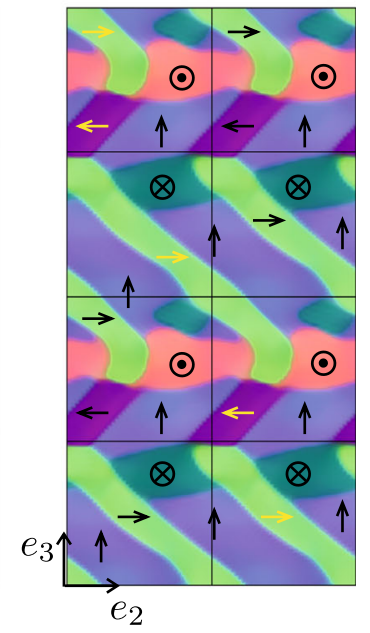

Fig. 10 Cross sections through the multi-cell assembly for ISSF and reverse periodic boundary condition along the $\mathbf{e}_{3}$ direction. a Showing the locations of the slices. $\mathbf{b}-\mathbf{d}$ Cross sections normal to: $\mathbf{b}$ the $\mathbf{e}_{3}$ direction, $\mathbf{c}$ the $\mathbf{e}_{2}$ direction, $\mathbf{d}$ the $\mathbf{e}_{1}$ direction

Further simulations using the isotropic spherical stress-free side condition (ISSF) yield similarly complex patterns, see Fig. 10. These domain patterns have many $180^{\circ}$ domain walls, along with ribbon-like domains [50] that thread through the structure. In both cases, five of the six polarization variants are present, which can be understood as follows: the strain condition enforces the presence of some domains with $c$ axis aligned to each of the three coordinate axes. However, polarization must balance in both the $\pm \mathbf{e}_{1}$ and $\pm \mathbf{e}_{2}$ directions due to the rotation of the cube during assembly, so all four variants polarized in the $\left(x_{1}, x_{2}\right)$ plane must be present. Meanwhile, since some domains with $c$ axis aligned to the $\mathbf{e}_{3}$ are expected at least five variants are likely to be present overall. From the methods employed by Tsou et al. [5,6], it is immediately evident that the pattern formed cannot be any laminate of rank less than 3 . The patterns shown appear similar to rank-3 laminates, but with imperfect alignment of domains and curved domain walls. The equilibrium domain patterns in Figs. 9 and 10 both exhibit centres of vorticity and ribbon-like domains, with five of the six polarization variants present. Close inspection reveals that they are related variants. This is reflected in their similar $\boldsymbol{\alpha}$ and $\boldsymbol{\beta}$ values (see Table 1).

The result of applying the adapted spherical stress-free case (ASSF) and reverse boundary condition on the $41 \mathrm{~nm}$ cube is the same as that shown in Fig. $3 \mathrm{~g}$ for the $33 \mathrm{~nm}$ cube. Thus, there is no need for an additional figure.

Finally, the result of applying the transversely isotropic deformation case (TID), along with the reverse periodic boundary condition, is shown in Fig. 11 . As expected, the formation of a $180^{\circ}$ domain wall is enforced. However, the result is as simple as possible: we observe alternating $180^{\circ}$ domain stripes, a well-known domain pattern in barium titanate. While the TID constraint with reverse periodic boundary condition also admits a single domain equilibrium state, it appears that the alternating stripes is a more likely end state because of the high probability that, during the phase transition, different regions in the periodic cell may nucleate domains aligned in different directions. As the simulation proceeds towards equilibrium, these regions grow and stabilize. There is then an energy barrier to be overcome if one region is to be eliminated to allow the pattern to become a monodomain. As with the standard periodic boundary condition, the equilibrium states computed with the reverse periodic boundary condition show a progression in free energy values, reducing in the order IFPT $\rightarrow$ TID $\rightarrow$ ISSF $\rightarrow$ ASSF, consistent with the gradual reduction of constraint from the most restrictive $(\bar{\varepsilon}=0)$ to the least restrictive, adaptive condition.

\subsection{Impact of an outer electric field}

Finally, we demonstrate the effect of an outer electric field on the stability of a 3D ribbon-like periodic domain pattern solution. As representative example, let us choose the polarization pattern modelled with 
(a)

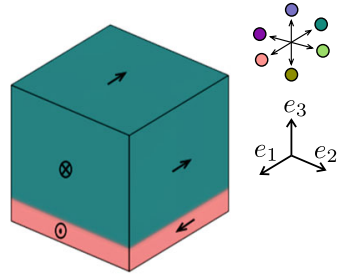

(b)

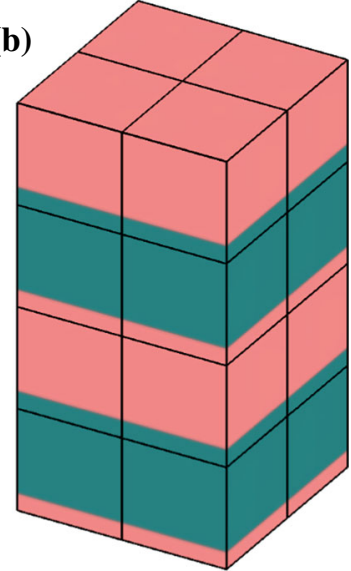

Fig. 11 Equilibrium domain pattern computed with the reverse periodic boundary condition along the $\mathbf{e}_{3}$ direction and the TID constraint. a The simulated volume. b An assembly of periodic cells

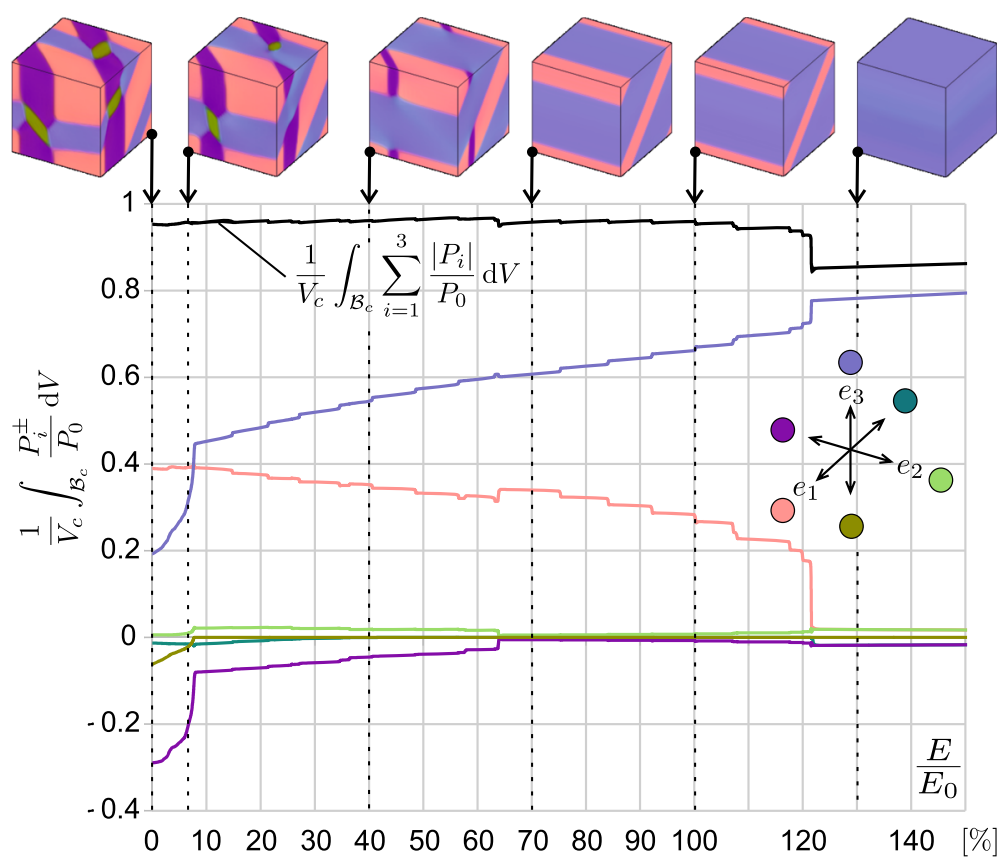

Fig. 12 Effect of the electric field along $+\mathbf{e}_{3}$-axis on the domain pattern modelled with isochoric constraint (IFPT) and standard periodic boundary conditions. The volume fractions of the six polarization variants are represented by equally coloured lines in the diagram. The black line represents the total amount of polarization in the RVE. Inset figures illustrate the evolving polarization pattern under the applied electric field. (Color figure online)

isochoric constraint (IFPT) and standard periodic boundary condition. The electric field is applied along the $+\mathbf{e}_{3}$ direction. In Fig. 12, the volume fractions of the six polarization variants in the RVE are plotted as coloured lines. The corresponding arrangements of the domains are shown as subset images at significant stages.

In the initial stages, up to $E=0.08 E_{0}$ the smaller domains with polarization in the $-\mathbf{e}_{3}$ direction shrink in size and disappear. However, the domains with polarization in $\mathbf{e}_{1}$ and $-\mathbf{e}_{2}$ direction remain such that the ribbon-like domain pattern is stable up to $E=0.64 E_{0}$, beyond which a striped domain pattern with $\mathbf{e}_{1}$ and $\mathbf{e}_{3}$ domains forms. On increasing the electric field up to $E=E_{0}$, the domains with polarization along $\mathbf{e}_{1}$ direction remain in the polarization pattern. We interpret this polarization pattern as arising from the competing isochoric mechanical side condition (IFPT) and the applied electric field. At $E=E_{0}$, the IFPT constraint stabilizes the $\mathbf{e}_{1}$-domain in the presence of opposing external field. 
However, at $E=1.21 E_{0}$, the stripe pattern dissolves to form a monodomain with net polarization along $\mathbf{e}_{3}$-axis. The net polarization in the domain pattern decreases during the transition from the stripe pattern to a monodomain. We associate this decrease in net polarization to result from the strong IFPT mechanical constraint. The mechanical strains modelled in the transverse direction to the applied electric field, force the monodomain away from its relaxed domain state.

\section{Conclusions}

In this work, we have applied multiple periodic boundary conditions for the simulation of ferroelectric domain patterns on the nanoscale. For the well-known standard conditions, our solutions tend to evolve patterns with $90^{\circ}$ domain walls only, if the RVE is allowed to adopt the inherent volumetric growth resulting from the materials phase transition from the cubic to the tetragonal crystal state. This result has been observed for three important variants (ASSF, ISSF, TID) of many more possible mechanical side conditions. However, we have also found a mechanical side condition enforcing $180^{\circ}$ domain walls. It restricts the RVE concerning its isochoric deformation, which we have denoted the isochoric ferroelectric phase transition (IFPT).

Since many experimental works do not restrict the isochoric deformation of the crystal during its ferroelectric phase transition, and since they have found ferroelectric patterns with $180^{\circ}$ domain walls, we have proposed the reverse periodic boundary condition, which naturally enforces $180^{\circ}$ domain walls within the RVE by an enhanced continuation rule of the cells. This extends the capabilities of our simulations and reproduces some domain patterns that are well known from prior theoretical studies and from observations of $\mathrm{BaTiO}_{3}$.

The reverse periodic boundary condition might be of interest for similar simulations in multiphysics; we just discuss one specific application here. But even for pure mechanical problems it widens the solution space of the displacement field. We refer to Fig. 8a, where the exaggerated deformation of the equilibrium state is deliberately shown. Obviously, displacement fields exist such that the assembly of cells requires the superposition of translation and rotation. To the best knowledge of the authors, the reverse periodic boundary condition is novel in the context of RVE and ferroelectric simulation models.

For both kinds of periodic boundary conditions, the simulations also exhibited interesting features such as frustrated domain walls, curved ribbon-like domains, and centres of vorticity. Some of these features can be of use in ferroelectric devices, and the simulations suggest that, by controlling strain during the cubic-ferroelectric transition, it may be possible to generate arrays of domains with these exotic features.

Open Access This article is distributed under the terms of the Creative Commons Attribution 4.0 International License (http:// creativecommons.org/licenses/by/4.0/), which permits unrestricted use, distribution, and reproduction in any medium, provided you give appropriate credit to the original author(s) and the source, provide a link to the Creative Commons license, and indicate if changes were made.

\section{References}

1. Devonshire, A.F.: Theory of barium titanate-Part I. Philos. Mag. Ser. 7 40(309), 1040-1063 (1949)

2. Devonshire, A.F.: Theory of barium titanate-Part II. Philos. Mag. J. Sci. 42(333), 1065-1079 (1951)

3. Cheng, S.Y., Ho, N.J., Lu, H.Y.: Transformation-induced twinning: the $90^{\circ}$ and $180^{\circ}$ ferroelectric domains in tetragonal barium titanate. J. Am. Ceram. Soc. 89(7), 2177-2187 (2006)

4. Hu, Y.H., Chan, H.M., Wen, Z.X., Harmer, M.P.: Scanning electron microscopy and transmission electron microscopy study of ferroelectric domains in doped $\mathrm{BaTiO}_{3}$. J. Am. Ceram. Soc. 69(8), 594-602 (1986)

5. Tsou, N.T., Potnis, P.R., Huber, J.E.: Classification of laminate domain patterns in ferroelectrics. Phys. Rev. B-Condens. Matter Mater. Phys. 83(184120), 1-6 (2011)

6. Tsou, N.T., Huber, J.E., Cocks, A.C.F.: Evolution of compatible laminate domain structures in ferroelectric single crystals. Acta Mater. 61, 670-682 (2013)

7. Arlt, G., Sasko, P.: Domain configuration and equilibrium size of domains in $\mathrm{BaTiO}_{3}$ ceramics. J. Appl. Phys. 51, $4956-4960$ (1980)

8. Jin, L., Xi, Z., Xu, Z., Yao, X.: Study of ferroelectric domain morphology in PMN-32\% PT single crystals. Ceram. Int. 30, $1695-1698(2004)$

9. Tagantsev, A.K., Cross, L.E., Fousek, J.: Domains in Ferroic Crystals and Thin Films. Springer, New York (2010)

10. Fousek, J., Safrankova, M.: On the equilibrium domain structure of $\mathrm{BaTiO}_{3}$. Jpn. J. Appl. Phys. 4, 403-408 (1965)

11. McGilly, L.J., Schilling, A., Gregg, J.M.: Domain bundle boundaries in single crystal $\mathrm{BaTiO}_{3}$ lamellae: searching for naturally forming dipole flux- closure/quadrupole chains. Nano Lett. 10(4200), 1-5 (2010) 
12. Roytburd, A.L., Alpay, S.P., Bendersky, L.A., Nagarajan, V., Ramesh, R.: Three-domain architecture of stress-free epitaxial ferroelectric films. J. Appl. Phys. 89, 553-556 (2001)

13. Renuka Balakrishna, A., Huber, J.E.: Scale effects and the formation of polarization vortices in tetragonal ferroelectrics. Appl. Phys. Lett. 106, 092906 (2015)

14. Balakrishna, A.R., Huber, J.E., Muench, I.: Periodic domain patterns in tetragonal ferroelectrics at the nanoscale-a phase field study. Phys. Rev. B 93(17), 1-13 (2016)

15. Hooton, J.A., Merz, W.J.: Etch patterns and ferroelectric domains in $\mathrm{BaTiO}_{3}$ single crystals. Phys. Rev. 98(2), 409-413 (1955)

16. Kontsos, A., Landis, C.M.: Phase-field modeling of domain structure energetics and evolution in ferroelectric thin films. J. Appl. Mech. 77, 041014 (2010)

17. Arlt, G.: The influence of microstructure on the properties of ferroelectric ceramics. Ferroelectrics 104(1), 217-227 (1990)

18. Arlt, G.: The role of domain walls on the dielectric, elastic and piezoelectricproperties of ferroelectric ceramics. Ferroelectrics 76(1), 451-458 (1987)

19. Shu, Y.C., Bhattacharya, K.: Domain patterns and macroscopic behaviour of ferroelectric materials. Philos. Mag. B 81(12), 2021-2054 (2001)

20. Muench, I., Huber, J.E.: A hexadomain vortex in tetragonal ferroelectrics. Appl. Phys. Lett. 95(022913), 1-3 (2009)

21. Rödel, J.: Effective intrinsic linear properties of laminar piezoelectric composites and simple ferroelectric domain structures. Mech. Mater. 39, 302-325 (2007)

22. Weng, G.J., Wong, D.T.: Thermodynamic driving force in ferroelectric crystals with a rank-2 laminated domain pattern, and a study of enhanced electrostriction. J. Mech. Phys. Solids 57, 571-597 (2009)

23. Scott, J.F.: Applications of modern ferroelectrics. Science 315(954), 1-9 (2007)

24. Renuka Balakrishna, A., Huber, J.E., Landis, C.M.: Nano-actuator concepts based on ferroelectric switching. Smart Mater. Struct. 23(8), 085016 (2014)

25. Krauß, M., Muench, I., Landis, C.M., Wagner, W.: Phase-field simulation and design of a ferroelectric nano-generator. Proc. SPIE 797821, 1-12 (2011)

26. Muench, I., Krauß, M., Landis, C.M., Huber, J.E.: Domain engineered ferroelectric energy harvesters on a substrate. J. Appl. Phys. 109(104106), 1-5 (2011)

27. Muench, I., Krauß, M., Wagner, W., Kamlah, M.: Ferroelectric nanogenerators coupled to an electric circuit for energy harvesting. Smart Mater. Struct. 21(115026), 1-8 (2012)

28. Li, Y.L., Hu, S.Y., Liu, Z.K., Chen, L.Q.: Phase-field model of domain structures in ferroelectric thin films. Appl. Phys. Lett. 78, $3878(2001)$

29. Wang, J., Shi, S.-Q., Chen, L.-Q., Li, Y., Zhang, T.-Y.: Phase-field simulations of ferroelectric/ferroelastic polarization switching. Acta Mater. 52(3), 749-764 (2004)

30. Chen, L.-Q.: Phase-field method of phase transitions/domain structures in ferroelectric thin films: a review. J. Am. Ceram. Soc 91(6), 1835-1844 (2008)

31. Woldman, A.Y., Landis, C.M.: Phase-field modeling of ferroelectric to paraelectric phase boundary structures in single-crystal barium titanate. Smart Mater. Struct. 25, 035033 (2016)

32. Schrade, D., Keip, M.-A., Thai, H., Schröder, J., Svendsen, B., Müller, R., Gross, D.: Coordinate invariant phase field modeling of ferroelectrics, Part I: model formulation and single crystal simulations. GAMM Mitteilungen 38, 102-114 (2015)

33. Wang, J.J., Ma, X.Q., Li, Q., Britson, J., Chen, L.-Q.: Phase transitions and domain structures of ferroelectric nanoparticles: phase field model incorporating strong elastic and dielectric inhomogeneity. Acta Mater. 61, 7591-7603 (2013)

34. Ouyang, J., Slusker, J., Levin, I., Kim, D.-M., Eom, C.-B., Ramesh, R., Roytburd, A.L.: Engineering of self-assembled domain architectures with ultra-high piezoelectric response in epitaxial ferroelectric films. Adv. Funct. Mater. 17(13), 2094-2100 (2007)

35. Li, Y.L., Hu, S.Y., Chen, L.Q.: Ferroelectric domain morphologies of (001) $\mathrm{PbZr}_{1-x} \mathrm{Ti}_{x} \mathrm{O}_{3}$ epitaxial thin films. J. Appl. Phys. 97(3), 034112 (2005)

36. Li, Y.L., Hu, S.Y., Liu, Z.K., Chen, L.Q.: Effect of substrate constraint on the stability and evolution of ferroelectric domain structures in thin films. Acta Mater. 50(2), 395-411 (2002)

37. Wang, J., Li, Y., Chen, L.Q., Zhang, T.Y.: The effect of mechanical strains on the ferroelectric and dielectric properties of a model single crystal-phase field simulation. Acta Mater. 53(8), 2495-2507 (2005)

38. Nahas, Y., Prokhorenko, S., Louis, L., Gui, Z., Kornev, I., Bellaiche, L.: Discovery of stable skyrmionic state in ferroelectric nanocomposites. Nat. Commun. 6, 8542 (2015)

39. Su, Y., Landis, C.M.: Continuum thermodynamics of ferroelectric domain evolution: theory, finite element implementation, and application to domain wall pinning. J. Mech. Phys. Solids 55, 280-305 (2007)

40. Kontsos, A., Landis, C.M.: Computational modeling of domain wall interactions with dislocations in ferroelectric crystals. Int. J. Solids Struct. 46, 1491-1498 (2009)

41. Devonshire, A.F.: Theory of ferroelectrics. Adv. Phys. 3(4), 85-130 (1954)

42. Fried, E., Gurtin, M.E.: Continuum theory of thermally induced phase transitions based on an order parameter. Physica D 68, 326-343 (1993)

43. Fried, E., Gurtin, M.E.: Dynamic solid-solid transitions with phase characterized by an order parameter. Physica D 72, 287-308 (1994)

44. Muench, I., Krauß, M.: An enhanced finite element technique for diffuse phase transition. Comput. Mech. 56, 691-708 (2015)

45. Krauß, M., Muench, I.: A selective enhanced FE-method for phase field modeling of ferroelectric materials. Comput. Mech. 57, 105-122 (2016)

46. Cao, W., Cross, L.E.: Theory of tetragonal twin structures in ferroelectric perovskites with a first-order phase transition. Phys. Rev. B 44, 5-12 (1991) 
47. Hlinka, J., Márton, P.: Phenomenological model of a $90^{\circ}$ domain wall in $\mathrm{BaTiO}_{3}$-type ferroelectrics. Phys. Rev. B 74, 104104 (2006)

48. Wang, J.J., Meng, F.Y., Ma, X.Q., Xu, M.X., Chen, L.Q.: Lattice, elastic, polarization, and electrostrictive properties of $\mathrm{BaTiO}_{3}$ from first-principles. J. Appl. Phys. 108(034107), 1-6 (2010)

49. McQuaid, R.G.P., McGilly, L.J., Sharma, P., Gruverman, A.: Mesoscale flux-closure domain formation in single-crystal $\mathrm{BaTiO}_{3}$. Nat. Commun. 2, 404 (2011)

50. Vorotiahin, I.S., Eliseev, E.A., Li, Q., Kalinin, S.V., Genenko, Y.A., Morozovska, A.N.: Tuning the polar states of ferroelectric films via surface charges and flexoelectricity. Acta Mater. 137, 85-92 (2017)

51. Li, Y.L., Chen, L.Q.: Temperature-strain phase diagram for $\mathrm{BaTiO}_{3}$ thin films. Appl. Phys. Lett. 88(7), 072905 (2006)

52. Haeni, J.H., Irvin, P., Chang, W., Uecker, R., Reiche, P., Li, Y.L., Choudhury, S., Tian, W., Hawley, M.E., Craigo, B., Tagantsev, A.K.: Room-temperature ferroelectricity in strained $\mathrm{SrTiO}_{3}$. Nature 430, 758-761 (2004)

53. Schlom, D.G., Chen, L.Q., Eom, C.B., Rabe, K.M., Streiffer, S.K., Triscone, J.M.: Strain tuning of ferroelectric thin films. Annu. Rev. Mater. Res. 37, 589-626 (2007)

54. Lu, H., Bark, C.W., De Los Ojos, D.E., Alcala, J., Eom, C.B., Catalan, G., Gruverman, A.: Mechanical writing of ferroelectric polarization. Science 336(6077), 59-61 (2012)

Publisher's Note Springer Nature remains neutral with regard to jurisdictional claims in published maps and institutional affiliations. 